\title{
Du forage au poste de soin : processus nouveau de distribution d'eaux hyperthermales à Luchon
}

\author{
J.F. Fries et R. Pigassou
}

Thermes municipaux de Luchon

\section{I $\square$ LUCHON : LA STATION}

Luchon, première station thermale de Midi-Pyrénées et première station ORL-Voies Respiratoires d'Europe traitant chaque saison (avril-octobre) environ 27000 curistes (ORL-VR et Rhumatologie), dispose d'eaux hyperthermales sulfurées-sodiques jaillissant à $74^{\circ} \mathrm{C}$ et $65^{\circ} \mathrm{C}$ ( 2 forages à 130-160 m). Depuis 1993-94 la distribution de ces eaux dans les divers Services de soins a été totalement modifiée.

\section{II — LE RÉSEAU DE DISTRIBUTION}

Le réseau de distribution de ces eaux thermales dans les trois bâtiments contigus des Thermes $\left(25000 \mathrm{~m}^{2}\right.$ de plancher) comporte 3500 vannes et robinets de tous types et de tous diamètres, répartis sur environ $15 \mathrm{~km}$ de canalisations en PVC et PVC Haute Température, de diamètres divers $(12 \mathrm{~mm}$ à $160 \mathrm{~mm})$, entrecoupés de 9 groupes de surpresseurs et de réservoirs maçonnés $\left(500 \mathrm{~m}^{3}\right)$ et bâches souples en néoprène armé $\left(250 \mathrm{~m}^{3}\right)$, pour alimenter, grâce à 17 échangeurs à plaques, les 1300 postes de soins ORL-VR et les 600 postes de soins Rhumatologie.

\section{L'ALIMENTATION DES POSTES DE SOINS}

\begin{abstract}
L'alimentation en eau thermale de tous les Services de soins a été complètement modifiée et réaménagée pour supprimer la quasi-totalité des risques de contamination inhérents aux eaux et à leur distribution. En effet, depuis 1991, l'eau thermale mise en œuvre dans les Thermes provient exclusivement des deux forages fournissant des eaux sulfurées sodiques à $65^{\circ} \mathrm{C}$ et $74^{\circ} \mathrm{C}$. Ces deux forages sont gainés en tubes d'acier inoxydable $(316 \mathrm{~L})$ jusqu'à une profondeur de 60 mètres pour éviter tout contact de ces eaux thermales avec les couches superficielles du sol, qui peuvent être polluées à partir de la surface, et pourraient contaminer les eaux thermales. A partir de ces forages, les deux eaux thermales ainsi disponibles (dénommées PRE et REINE) sont conduites à température nominale $\left(65^{\circ} \mathrm{C}\right.$ et $74^{\circ} \mathrm{C}$ ) jusqu'à la porte des salles de soins, où elles sont alors refroidies à température d'utilisation par des échangeurs à plaques. Les conduites et les réservoirs intermédiaires sont donc tous parcourus par de l'eau dont la température est supérieure à $60^{\circ} \mathrm{C}$, ce qui élimine tout risque de pollution ou contamination.
\end{abstract}

\section{From the borehole to the treatment room : a new process for mineral water distribution in Luchon}

Luchon has springs of sulphur and soda-rich mineral waters emerging at $74^{\circ} \mathrm{C}$ and $65^{\circ} \mathrm{C}$. Since $1993-94$ the way in which these waters are distributed to the various treatment rooms has been completely modified: to eliminate any risk of contamination, the boreholes are partially lined with stainless steel and the water is kept at nominal temperature throughout the transit and intermediate storage systems. It is only at the very door of the treatment rooms that the water is cooled to its temperature of use by means of plate heat exchangers.

The paper describes this completely new process, highlighting the technical requirements and problems which have had to be resolved together with the safety systems such an installation requires. 
En outre, pour tous les Services de soins ORL-VR, les eaux thermales transitent par des bâches souples étanches, qui se gonflent et se dégonflent au gré des consommations, afin d'empêcher tout contact de l'eau avec l'atmosphère, supprimant ainsi tout risque de contamination extérieure et toute transformation des sulfures de l'eau en sulfates par réaction chimique avec l'oxygène de l'air. Cette installation technique totalement nouvelle constitue un avantage décisif pour les Thermes de Luchon, et a été très appréciée par les Services de la DDASS.

\section{D LA SÉCURITÉ}

L'acheminement de l'eau hyperthermale à température nominale dans les circuits de distribution posait néanmoins un grave problème de sécurité. En effet, la défaillance technique du système de refroidissement est toujours possible, même si elle est très improbable dans une installation entretenue et contrôlée régulièrement et méticuleusement. En pareille circonstance, il faut absolument exclure toute possibilité que le curiste puisse être en contact avec une eau thermale à température supérieure à $60^{\circ} \mathrm{C}$. C'est pourquoi toutes les canalisations de sortie des échangeurs à plaques ont été équipées d'une vanne automatique de sécurité, thermiquement activée par toute arrivée d'eau à température du soin (soit 34 à $42^{\circ} \mathrm{C}$ selon les échangeurs). D'autre part des points de prélèvement pour analyses de contrôle des eaux ont été installés dans tout le réseau : aux captages, à l'entrée et à la sortie de tous les réservoirs, à chaque entrée et sortie d'échangeur et sur les postes de soins ORL. Ces prélèvements sont réalisés à l'aide de becs inox.

\section{V —LA DÉSINFECTION}

Ce système permet de pratiquer une désinfection par portion de réseau au moyen de désinfectant (utilisé en sanitation des circuits dans les industries alimentaires des produits d'origine animale ou végétale et les embouteillages d'eau) à base de péroxyde d'hydrogène-acide péracétique.

\section{CONCLUSION}

Compte tenu de l'ensemble de ces aménagements très importants, réalisés durant deux inter-saisons successives, Luchon possède maintenant une installation de distribution des eaux thermales à la pointe des nécessités sanitaires, en rapport avec sa dimension et sa renommée européenne. 